\title{
IMPORTANCE OF METHODOLOGY IN DETERMINING BACTERICIDAL AND BACTERIOSTATIC ACTIVITIES OF AZLOCILLIN AND TICARCILLIN AGAINST PSEUDOMONAS AERUGINOSA
}

\author{
W. Brumfitt, J. M. T. Hamilton-Miller and A. Gooding \\ Department of Medical Microbiology, Royal Free Hospital and Medical School, \\ Pond Street, London NW3 $2 Q G$
}

\begin{abstract}
Summary. The activities of azlocillin and ticarcillin against Pseudomonas aeruginosa were compared by estimating minimum inhibitory and bactericidal concentrations (MIC and $\mathrm{MBC}$ ) in liquid and solid media, and by constructing killing curves from sequential viable counts. In MIC studies, azlocillin was about three times more active than ticarcillin in solid medium (agar dilution test) and in liquid media (tube and microdilution tests). When the MBC was measured, however, results varied according to the technique used. On agar and in microdilution tests, both azlocillin and ticarcillin were bactericidal, the $\mathrm{MBC}$ being 1.3-3 MIC. In the tube test, the $\mathrm{MBC}$ for ticarcillin was again about $3 \mathrm{MIC}$, but azlocillin appeared not to be bactericidal (MBC $>1 \mathrm{mg} / \mathrm{ml}$ ). However, sequential viable counts of four clinical isolates showed that at $4 \mathrm{MIC}$ both antibiotics reduced viable counts by a factor of $10^{4}$ in $8 \mathrm{~h}$. Our results stress the importance of methodology when assessing the antibacterial activity of an antibiotic.
\end{abstract}

\section{INTRODUCTION}

Pseudomonas aeruginosa most often causes opportunistic infections in compromised patients, and effective treatment must be instituted at once to avoid a high mortality rate. P. aeruginosa is not only intrinsically resistant to many antibiotics, but is also able to acquire resistance to others with relative ease. Hence, the search for compounds with antipseudomonal activity has continued. Azlocillin is a ureidopenicillin which has recently been marketed in Great Britain as an alternative to the well-established compound ticarcillin; it is currently in use in this Hospital. Because suggestions have been made that azlocillin has certain shortcomings in respect of its activity against $P$. aeruginosa (Basker, Edmondson and Sutherland, 1979; White, Comber and Sutherland, 1980), we have undertaken a detailed study of a large number of freshly isolated clinical strains.

Different techniques for measuring the minimum inhibitory concentration (MIC) and the minimum bactericidal concentration $(\mathrm{MBC})$ were used, in view of the findings 
of Greenwood and Eley (1982) that conventional MIC tests and turbidimetric experiments gave different results when used for assessing the activity of $\beta$-lactam antibiotics against $P$. aeruginosa.

\section{MATERIALS AND METHODS}

Media. Oxoid Iso-Sensitest Broth (ISB) and Iso-Sensitest Agar (ISA) were used throughout this study.

Organisms tested. Two series of $P$. aeruginosa strains were used. Series A consisted of 110 consecutive isolates obtained recently from clinical material sent to the Diagnostic Microbiology Laboratory at the Royal Free Hospital. Series B consisted of 18 gentamicin-resistant strains collected during a 2-year period from clinical specimens. These strains were epidemiologically distinct; multiple isolates from the same patient were excluded and there were no recorded instances of cross-infection with these strains. These 128 strains were tested for sensitivity by the disk diffusion method (see below). Further experiments were performed with strains selected randomly from the 95 strains in series A that were sensitive to both azlocillin and ticarcillin.

Disk sensitivity tests. Strains were cultured overnight in digest broth, diluted 1 in $10^{4}$ with water, and $0 \cdot 1-\mathrm{ml}$ amounts were spread on plates of ISA to give an inoculum of $c .10^{4} \mathrm{cfu} / \mathrm{plate}$. Disks containing azlocillin $(75 \mu \mathrm{g})$, ticarcillin $(75 \mu \mathrm{g})$ and gentamicin $(10 \mu \mathrm{g})$ were placed on the plates, which were then incubated overnight. Strains that gave zones of inhibition of $13 \mathrm{~mm}$ or less were regarded as resistant. This is consistent with the criteria laid down by Brown and Blowers (1978) for gentamicin, Phaneuf and Neu (1979) for ticarcillin and Fass (1982) for azlocillin. Disks were controlled with a standard sensitive strain, $P$. aeruginosa NCTC10662.

\section{Determination of $M I C$ and $M B C$}

Agar dilution method. Serial doubling dilutions of azlocillin and ticarcillin in ISA were inoculated with $10^{4}, 10^{5}$ and $10^{6} \mathrm{cfu}$ of 82 strains of $P$. aeruginosa, using a multipoint inoculator (Denly Instruments, Billinghurst, Sussex RH14 9SJ). MICs were read after incubation for $18 \mathrm{~h}$ at $37^{\circ} \mathrm{C}$; MBCs were determined by replica plating with a velvet pad from the surface of the plates inoculated with $10^{5} \mathrm{cfu}$ to fresh ISA, and incubating overnight. Because the efficiency of transfer with the velvet pad is $c .1 \%$ (Cruickshank, Duguid and Swain, 1969), a $99.8 \%$ fall in viable count was represented by the growth of two colonies after this procedure.

Broth dilution method. Serial doubling dilutions of azlocillin and ticarcillin were made in $1-\mathrm{ml}$ amounts of ISB contained in soda lime glass tubes $(7.5 \mathrm{~cm} \times 1 \mathrm{~cm})$. Tubes were inoculated with $10^{5} \mathrm{cfu}$ of $P$. aeruginosa $\left(9\right.$ strains) and the MIC was read after incubation overnight at $37^{\circ} \mathrm{C}$. The $\mathrm{MBC}$ was determined by sub-culturing $5 \mu \mathrm{l}$, with a Nichrome loop of $3.5 \mathrm{~mm}$ diameter, on to blood agar from each tube in which no growth was visible. The growth of one colony indicated a $99.8 \%$ fall in viable count.

Microdilution method. Serial doubling dilutions of azlocillin and ticarcillin were made in $50-\mu \mathrm{l}$ amounts of ISB containing triphenyltetrazolium chloride $200 \mu \mathrm{g} / \mathrm{ml}$ in Microtiter "V" plates made of tissue culture grade polystyrene. Each well was inoculated with $150 \mu \mathrm{l}$ of ISB that contained $4 \times 10^{5} \mathrm{cfu}$ of $P$. aeruginosa; 45 strains were tested. Plates were stacked to avoid evaporation and incubated overnight at $37^{\circ} \mathrm{C}$. In the wells where growth had occurred the tetrazolium salt was reduced to a red formazan and the appearance of the red colour was used to determine the MIC end-point. The MBC was established by sub-culturing $1 \mu \mathrm{l}$ from each well of every plate on to ISA, using a multipoint inoculator equipped with a 96-pin head. The growth of four or fewer colonies indicated a $99.8 \%$ or greater fall in viable count.

MIC was the lowest concentration of antibiotic that prevented visible growth overnight (agar and tube dilution methods), or prevented reduction of tetrazolium overnight (microdilution method). $\mathrm{MBC}$ was the lowest concentration of antibiotic that caused at least a $99.8 \%$ fall in viable count during overnight incubation. Although the cut-off points for $\mathrm{MBC}$ were slightly different for the three techniques used, there was usually no problem in deciding the MBC 
because sub-cultures yielded either no growth, which by all three techniques indicated at least $99.9 \%$ fall in viable count, or a heavy growth.

Sequential viable counts. An inoculum of $10^{7} \mathrm{cfu}$ of each of four test strains was added to a series of five $250-\mathrm{ml}$ conical flasks each containing $100 \mathrm{ml}$ of ISB to which azlocillin or ticarcillin had been added to achieve concentrations representing 0.5 MIC, $1 \mathrm{MIC}, 2 \mathrm{MIC}$ and $4 \mathrm{MIC}$. An antibiotic-free control was also included. Flasks were swirled at $100 \mathrm{rpm}$ at $37^{\circ} \mathrm{C}$ and at suitable intervals $0 \cdot 1-\mathrm{ml}$ amounts were removed from each flask for a viable count on ISA containing penicillinase (Wellcome Laboratories, Beckenham, Kent BR3 3BS) at a dilution of 1 in 250.

Assay for antibiotic destruction. A total of 49 strains of $P$. aeruginosa (44 sensitive and 5 resistant to azlocillin, as judged by the disk test) were cultured in $2 \cdot 5-\mathrm{ml}$ amounts of broth at $37 \mathrm{C}$ overnight. An equal volume of broth containing azlocillin $1 \mathrm{ng} / \mathrm{ml}$ was then added to each bottle; incubation was continued for $6 \mathrm{~h}$ and the amount of intact antibiotic in each bottle was estimated by the hydroxylamine assay (Batchelor et al., 1961). Suitable controls were set up to ensure that any breakdown of azlocillin was not due to non-specific causes.

\section{RESULTS}

\section{Disk sensitivity testing}

In series A ( 110 consecutive isolates) all the strains were sensitive to gentamicin, and 95 were also sensitive to ticarcillin and azlocillin. Five strains were resistant to both ticarcillin and azlocillin; nine were resistant to ticarcillin but sensitive to azlocillin; one was resistant to azlocillin but sensitive to ticarcillin. Thus, six strains $(5 \cdot 5 \%)$ were resistant to azlocillin and $14(12.7 \%)$ to ticarcillin.

In series B (18 gentamicin-resistant strains), 10 strains were sensitive to ticarcillin and azlocillin, six were resistant to ticarcillin but sensitive to azlocillin, and two were resistant to both penicillins. The incidence of resistance to ticarcillin in gentamicinresistant strains was significantly higher $(\mathrm{P}<0.001)$ than in gentamicin-sensitive organisms. There was no significant difference in resistance to azlocillin in the two groups.

Cross-resistance between ticarcillin and azlocillin was not total; whereas $7(32 \%)$ of the 22 ticarcillin-resistant strains were sensitive to azlocillin, $7(88 \%)$ of the 8 azlocillin-resistant strains were resistant to ticarcillin.

\section{$M I C$ and $M B C$}

Geometric mean values of MIC and MBC for the two penicillins against $P$. aeruginosa strains are shown in the table. Azlocillin was 3-4 times more active than ticarcillin and there was close agreement between MICs determined by the three methods. However, a major discrepancy appeared when MBCs were considered. When tested by the agar dilution and microdilution methods, the mean MBC was 1.3-3 times greater than MIC for both compounds. The MBC for the great majority of strains was either identical to, or double, the MIC. In the tube dilution test, however, although a similar result was obtained with ticarcillin $(\mathrm{MBC}=3 \mathrm{MIC})$, azlocillin appeared not to be bactericidal (MBC $>1024 \mu \mathrm{g} / \mathrm{ml})$.

\section{Inoculum size}

The effect on MIC determined by the agar dilution technique of increasing the 
TABLE

Antibacterial activity of ticarcillin and azlocillin against $P$. aeruginosa, measured in different ways

\begin{tabular}{|c|c|c|c|c|c|c|}
\hline \multirow[b]{3}{*}{ Method } & \multirow{3}{*}{$\begin{array}{l}\text { Number of } \\
\text { strains }\end{array}$} & \multirow{3}{*}{$\begin{array}{l}\text { Inoculum } \\
\text { (cfu) }\end{array}$} & \multicolumn{4}{|c|}{ Antibacterial activity of } \\
\hline & & & \multicolumn{2}{|c|}{ Ticarcillin } & \multicolumn{2}{|c|}{ Azlocillin } \\
\hline & & & $\mathrm{MIC}$ & $\mathrm{MBC}$ & MIC & $\mathrm{MBC}$ \\
\hline Agar dilution & 82 & $10^{5}$ & $40 \cdot 5$ & $54 \cdot 4$ & $8 \cdot 7$ & $15 \cdot 5$ \\
\hline Tube dilution & 9 & $10^{5} / \mathrm{ml}$ & 40 & 118 & $6 \cdot 3$ & $>1024$ \\
\hline Microdilution & 45 & $4 \times 10^{5} /$ well & $38 \cdot 5$ & $100 \cdot 5$ & $9 \cdot 7$ & $26 \cdot 2$ \\
\hline
\end{tabular}

$\mathrm{MIC}=$ minimum inhibitory concentration $; \mathrm{MBC}=$ minimum bactericidal concentration; all values are geometric means, expressed as $\mu \mathrm{g} / \mathrm{ml}$.

inoculum from $10^{4}$ to $10^{6} \mathrm{cfu}$ was small; mean values of MIC increased only $1 \cdot 5-$ and 2-fold, respectively, for ticarcillin and azlocillin.

\section{Sequential viable counts}

The MICs of ticarcillin and azlocillin for the four strains used in these experiments ranged from 16 to $64 \mu \mathrm{g} / \mathrm{ml}$ and 2 to $16 \mu \mathrm{g} / \mathrm{ml}$ respectively. The results of sequential viable counts of these strains are shown in the figure. Ticarcillin was somewhat more bactericidal than azlocillin at concentrations of $1 \mathrm{MIC}$ and $2 \mathrm{MIC}$, but there was no difference in activity at the higher concentration of $4 \mathrm{MIC}$ : viable counts were lowered by a factor of approx. $10^{4}$ in $8 \mathrm{~h}$ by either antibiotic.

When cultures were examined $24 \mathrm{~h}$ after the start of the experiment, some regrowth had occurred with three of the four strains in the presence of $2 \mathrm{MIC}$ and $4 \mathrm{MIC}$ of azlocillin and with two of the four strains in the presence of $2 \mathrm{MIC}$ and $4 \mathrm{MIC}$ of ticarcillin.

\section{Azlocillin destruction by resistant strains}

All five azlocillin-resistant strains of $P$. aeruginosa tested destroyed azlocillin rapidly; in the test conditions, all the antibiotic added was hydrolysed within $6 \mathrm{~h}$. Rapid destruction did not occur with the 44 sensitive strains tested.

\section{Discussion}

Azlocillin, to which $94.5 \%$ of 110 consecutive clinical isolates of $P$. aeruginosa were sensitive when tested by the disk diffusion method, appeared more active than ticarcillin against this species. Furthermore, certain strains resistant to ticarcillin were sensitive to azlocillin. There was an increased incidence of resistance to ticarcillin, but not to azlocillin, amongst gentamicin-resistant strains. The reason for this is unclear. Resistance to these penicillins in P. aeruginosa is usually associated with the presence of plasmid-mediated TEM (class III) or PSE (class V) $\beta$-lactamases (Furth, 1979). All the azlocillin-resistant strains tested caused rapid and complete destruction of azlocillin. In our test conditions, sensitive strains did not destroy azlocillin rapidly, 


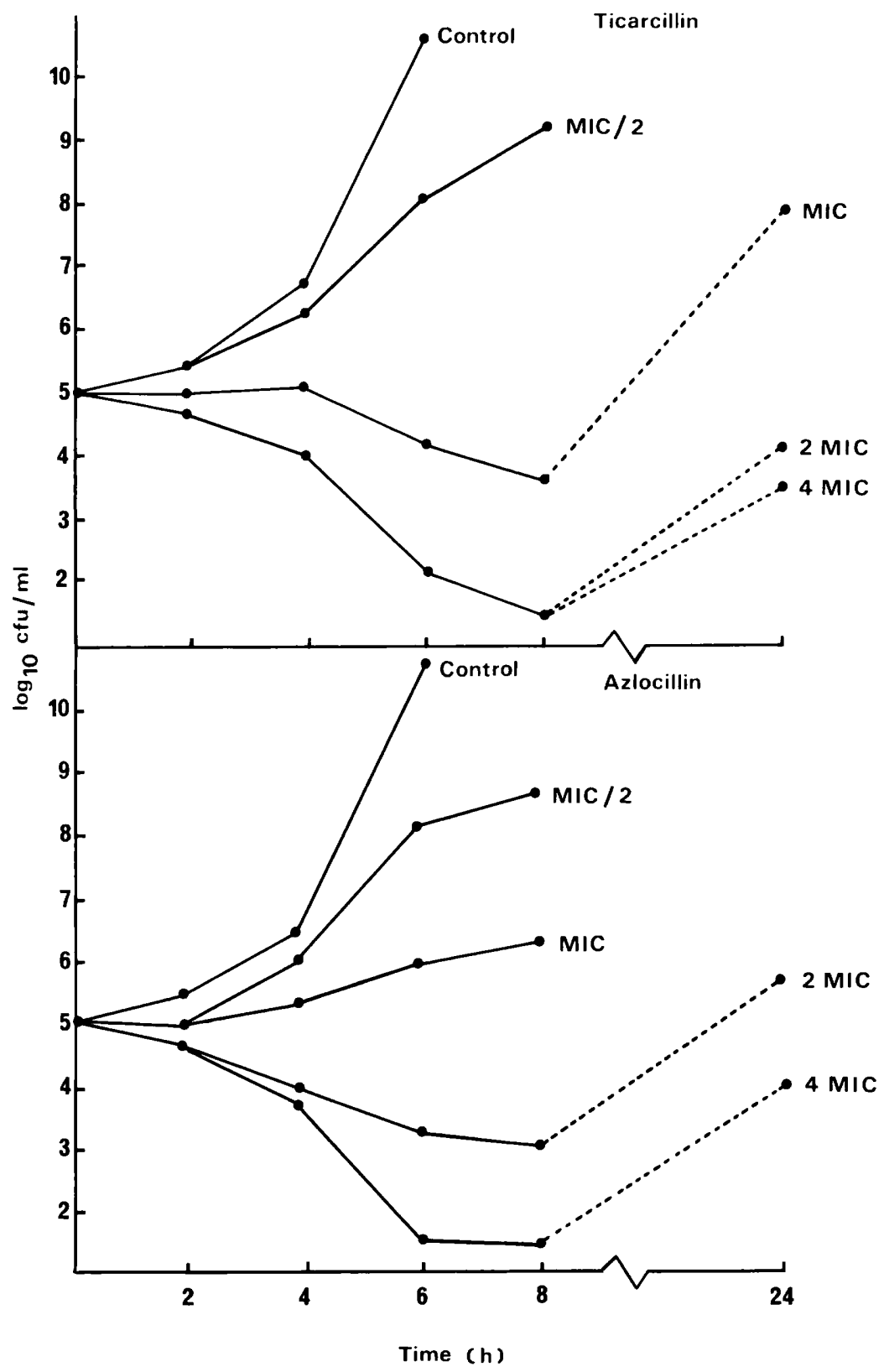

FIG.-Sequential viable counts of $P$. aeruginosa exposed to ticarcillin (upper) or azlocillin (lower) in Iso-sensitest broth. Each point is the mean obtained by testing four different strains. 
although other workers (Basker et al., 1979; Gwynn and Rolinson, 1980; White et al., 1980 ) have shown that the chromosomal $\beta$-lactamase (class Id) produced by virtually all $P$. aeruginosa strains hydrolyses azlocillin slowly. Ticarcillin is more stable to the Id enzyme than is azlocillin, but is equally susceptible to the class III and V enzymes (Labia et al., 1982).

The lability of azlocillin to the Id enzyme may explain our findings and those of other workers (Basker et al., 1979; Gwynn and Rolinson, 1980; Greenwood and Eley, 1982) that regrowth sometimes occurs when $P$. aeruginosa strains are cultured in concentrations of ureidopenicillins that are initially bactericidal. However, while recognising the phenomenon, we do not regard it as being necessarily of any relevance clinically, because in this type of experiment only a single dose of the penicillin is used, whereas in therapeutic conditions an 8-hourly dosage schedule is used.

There have been several suggestions that the ureidopenicillins may be deficient in bactericidal activity, especially against $P$. aeruginosa (Kurtz, Holmes and Turck, 1975; Fu and Neu, 1978; Shah et al., 1979). The present study shows that methodology may play a decisive role in whether azlocillin is judged to be bactericidal or not. By three of the four methods used, azlocillin and ticarcillin were approximately equivalent in their bactericidal action; only in liquid medium in static glass tubes did azlocillin appear to be poorly bactericidal. We regard the results of the experiments in which bacteria were shaken in broth in the presence of antibiotic and viable counts made at intervals as being the most reliable indication of bactericidal activity. In these conditions, both penicillins at concentrations of $2 \mathrm{MIC}$ and $4 \mathrm{MIC}$ were progressively bactericidal during a period of $8 \mathrm{~h}$. A concentration of $4 \mathrm{MIC}$ ranged from 8 to $64 \mu \mathrm{g} / \mathrm{ml}$ for azlocillin - readily attainable when 2 gm is given intravenously (Wirth, Schomerus and Hengstmann, 1976)-whereas to achieve equivalent concentrations of ticarcillin $(64-256 \mu \mathrm{g} / \mathrm{ml})$ a dosage of $3-5 \mathrm{~g}$ is required.

Some previous comparative studies on the antipseudomonal activity of azlocillin and ticarcillin or carbenicillin (Gwynn and Rolinson, 1980; White et al., 1980; Gwynn, Webb and Rolinson, 1981) have used standard laboratory strains such as $P$. aeruginosa NCTC10662 (synonymous with ATCC27853 and ATCC25668) as test organisms. Such strains do not necessarily represent fresh clinical isolates. We believe that more meaningful information can be obtained by the use of large numbers of unselected fresh clinical isolates.

Alpha-carboxypenicillins and ureidopenicillins both bind strongly to penicillinbinding protein 3 (PBP3) (Curtis et al., 1979). This results in extensive filamentation of the bacteria (Gwynn et al., 1981; Greenwood and Eley, 1982). However, the detailed modes of action of these two groups of penicillins are not identical, because $x$-carboxypenicillins, but not ureidopenicillins, also bind strongly to PBPla. Although the production of filaments causes bacteria to die less rapidly than a mode of action which brings about spheroplast formation and rapid lysis, antibiotics that cause filamentation are nevertheless effective in vitro (Curtis, 1981) and in experimental animals (Ryan and Monsey, 1981). Further evidence of their efficacy is that azlocillin and ticarcillin have excellent therapeutic records (O'Grady, 1978; Siegenthaler and Weuta, 1980), although in the compromised host-patients with malignancies, or leukopenic subjects - combination therapy with an aminoglycoside is preferred (EORTC International Antimicrobial Therapy Project Group, 1978).

Despite the similarities in overall action between azlocillin and ticarcillin, we did 
observe a diminished bactericidal activity of azlocillin in a liquid medium when the test was done in static glass tubes. This phenomenon has also been reported by Kurtz et al. (1975) and Fu and Neu (1978). However azlocillin was bactericidal in broth contained in microdilution trays. An obvious difference is that glass is a wettable surface but polystyrene (from which the microdilution trays were made) is not. If filamentous bacteria adsorb on to a glass surface they may be protected in some way from the bactericidal action of azlocillin, but not from that of ticarcillin. In vigorously shaken cultures adsorption would be markedly decreased, which would account for the bactericidal action observed by sequential viable counting. Gwynn et al. (1981) have also suggested that adsorption on to glass may protect $P$. aeruginosa from the bactericidal activity of penicillins.

In summary, we have shown, like Greenwood and Eley (1982), that different methods of assessing antimicrobial activity may give different results when $P$. aeruginosa is the test organism. It would be interesting to know if $P$. aeruginosa is unique in this respect, or if susceptibility testing of all organisms should be done by several methods. The question also remains as to which method of testing gives the information that is most clinically relevant.

\section{REFERENCES}

Basker M J, Edmondson R A E, Sutherland R 1979 Comparative antibacterial activity of azlocillin, mezlocillin, carbenicillin and ticarcillin and relative stability to beta-lactamases of Pseudomonas aeruginosa and Klebsiella aerogenes. Infection 7:67-73.

Batchelor F R, Chain E B, Hardy T L, Mansford K R L, Rolinson G N 1961 6-aminopenicillanic acid. III. Isolation and purification. Proceedings of the Royal Society B 154:498-508.

Brown D, Blowers R 1978 Disc methods of sensitivity testing and other semiquantitative methods. In: Reeves D S, Phillips I, Williams J D, Wise R (eds) Laboratory Methods in Antimicrobial Chemotherapy. Churchill Livingstone, Edinburgh, pp 8-30.

Cruickshank R, Duguid J P, Swain R H A 1969 Medical Microbiology, 11th edn. E. \& S. Livingstone, Edinburgh, $\mathrm{p} 901$.

Curtis N A C 1981 Penicillin-binding proteins in theory and practice. Journal of Antimicrobial Chemotherapy 8:85-87.

Curtis N A C, Orr D, Ross G W, Boulton M G 1979 Affinities of penicillins and cephalosporins for the penicillin-binding proteins of Escherichia coli $\mathrm{K} 12$ and their antibacterial activity. Antimicrobial Agents and Chemotherapy 15:533-539.

EORTC International Antimicrobial Therapy Project Group 1978 Three antibiotic regimens in the treatment of infection in febrile granulocytopenic patients with cancer. Journal of Infectious Diseases 137:14-29.

Fass R J 1982 Quality control and interpretive criteria for the azlocillin disk diffusion susceptibility test. Antimicrobial Agents and Chemotherapy 22:28-31.

Fu K P. Neu H C 1978 Azlocillin and mezlocillin: new ureido penicillins. Antimicrobial Agents and Chemotherapy 13:930-938.

Furth A 1979 The $\beta$-lactamases of Pseudomonas aeruginosa. In: Hamilton-Miller J M T, Smith J T (eds) Beta-lactamases. Academic Press, London, pp 403-428.

Greenwood D, Eley A 1982 A turbidimetric study of the responses of selected strains of Pseudomonas aeruginosa to eight antipseudomonal $\beta$-lactam antibiotics. Journal of Infectious Diseases 145:110-117.

Gwynn M N, Rolinson G N 1980 Selection of variants of Pseudomonas aeruginosa resistant to beta-lactam antibiotics. Infection 8:73-80.

Gwynn M N, Webb L T, Rolinson G N 1981 Regrowth of Pseudomonas aeruginosa and other bacteria after the bactericidal action of carbenicillin and other $\beta$-lactam antibiotics. Journal of Infectious Diseases 144:263-269. 
Kurtz S, Holmes K, Turck M 1975 Disparity between inhibitory and killing effects of BL-P1654 Antimicrobial Agents and Chemotherapy 7:215-218.

Labia R, Beguin R, Masson J M, Kazmierczak A 1982 Cefsulodin and Pseudomonas aeruginosa: the influence of $\beta$-lactamases. In: Periti P, Grassi G G (eds) Current chemotherapy and immunotherapy, 1. American Society of Microbiology, Washington, pp 511-513.

O'Grady F (editor) 1978 Ticarcillin (BRL 2288). Proceedings of an international symposium. Excerpta Medica, Amsterdam, International Congress Series no 445.

Phaneuf D, Neu H C 1979 Agar disk diffusion susceptibility characteristics of azlocillin, carbenicillin, mezlocillin, piperacillin and ticarcillin. Antimicrobial Agents and Chemotherapy 16:625-630.

Ryan D M, Monsey D 1981 Bacterial filamentation and in-vivo efficacy: a comparison of several cephalosporins. Journal of Antimicrobial Chemotherapy 7:57-63.

Shah P P, Briedis D J, Robson H G, Conterato J P 1979 In vitro activity of piperacillin compared with that of carbenicillin, ticarcillin, ampicillin, cephalothin and cefamandole against Pseudomonas aeruginosa and Enterobacteriaceae. Antimicrobial Agents and Chemotherapy 15:346-350.

Siegenthaler W, Weuta H (eds) 1980 Acylureidopenicillins. Proceedings of an international symposium. Excerpta Medica, Amsterdam.

White A R, Comber K R, Sutherland R 1980 Comparative bactericidal effects of azlocillin and ticarcillin against Pseudomonas aeruginosa. Antimicrobial Agents and Chemotherapy 18:182-189.

Wirth K, Schomerus M, Hengstmann J H 1976 Zur Pharmakokinetik von Azlocillin, einem neuen halbsynthetischen Breitspektrumantibiotikum Infection, 4:25-30. 